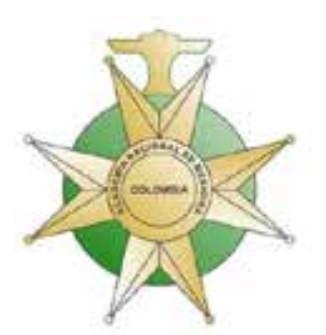

\title{
Recomendaciones de la Academia Nacional de Medicina de Colombia para enfrentar los conflictos éticos secundarios a la crisis de COVID-19 en el inicio y mantenimiento de medidas de soporte vital avanzado
}

\author{
Recommendations of the National Academy of Medicine of Colombia to \\ face the ethical conflicts secondary to the COVID-19 crisis in the initiation \\ and maintenance of advanced life support measures
}

\section{Equipo de trabajo:}

Ramón Abel Castaño Yepes ${ }^{1,2} \mathbb{D}$, Gloria Arias Nieto ${ }^{2,3}$, Oswaldo A. Borráez Gaona ${ }^{2,4} \mathbb{D}$, Alejandro Niño Murcia ${ }^{2,5} \mathbb{D}$, Liliana Támara Patiño ${ }^{2,6} \mathbb{D}$, Julieta Moreno Molina ${ }^{7}$

MD, Consultor en sistemas de salud, Bogotá, D.C., Colombia.

2 Miembro correspondiente de la Academia Nacional de Medicina.

3 MD, especialista en Bioética y en Administración de salud; magíster en Salud mental, Bogotá, D.C., Colombia.

4 MD, especialista en Cirugía General; expresidente Asociación Colombiana de Cirugía; presidente de Tribunal de Ética Médica de Bogotá, Bogotá, D.C., Colombia.

5 MD, especialista en Cirugía de Trasplantes, Colombiana de Trasplantes; presidente del Comité de Trasplantes de la Asociación Colombiana de Cirugía, Bogotá, D.C., Colombia.

6 MD, especialista en Bioética; candidata a magíster en Protección Social; Directora Comisión de Ética, Instituto Colombiano de Estudios Bioéticos, Bogotá, D.C., Colombia.

7 MD, magíster en Bioética; consultora Ministerio de Salud y Protección Social, Bogotá, D.C., Colombia.

Palabras clave: COVID-I9; virus del SRAS; coronavirus; infecciones por coronavirus; pandemias; ética médica; recursos en salud.

Key words: COVID-I9; SARS virus; coronavirus; coronavirus infections; pandemics; ethics, medical; health resources.

Fecha de recibido: 19/05/2020 - Fecha de aceptación: 28/05/2020 - Fecha de actualización: 10/07/2020

Correspondencia: Oswaldo A. Borraez G. Academia Nacional de Medicina, Carrera 7 No. 69-11, Bogotá, D.C., Colombia. Teléfono: 313-3350394. Correo electrónico: oborraezg@gmail.com

Citar como: Castaño Yepes RA, Arias Nieto G, Borráez Gaona O, Nino Murcia A, Támara Patiño L, Moreno Molina J. Recomendaciones de la Academia Nacional de Medicina de Colombia para enfrentar los conflictos éticos secundarios a la crisis de COVID-19 en el inicio y mantenimiento de medidas de soporte vital avanzado. Rev Colomb Cir. 2020;35:351-62. https://doi.org/10.30944/20117582.725

Este es un artículo de acceso abierto bajo una Licencia Creative Commons - BY-NC-ND https://creativecommons.org/licenses/by-ncnd/4.0/deed.es 


\section{Contexto}

La profesión médica, fiel a su compromiso de buscar el beneficio de los pacientes y lograr los mejores resultados posibles en el proceso de atención, respetando sus derechos y voluntad, se enfrenta al reto de la pandemia de COVID-I9 y la consecuente situación de emergencia sanitaria, la cual conlleva un estado de excepcionalidad que modifica de manera fundamental algunos elementos tradicionales de la práctica médica.

La infección por el virus SARS-CoV2 y la enfermedad causada por este, denominada COVID-I9, es una condición nueva para el conocimiento médico. En consecuencia, la evidencia científica crece rápidamente y se modifica con mayor frecuencia de lo habitual. A pesar de ello, a la fecha de elaboración del presente documento aún no se cuenta con una vacuna o con evidencia suficiente acerca de tratamientos farmacológicos específicos para el virus; sin embargo, sí se dispone de herramientas de diagnóstico, manejo de síntomas, soporte vital y paliación.

En lo concerniente al soporte vital, específicamente la atención en Unidades de Cuidados Intensivos (UCI), $\mathrm{u}$ otras medidas de soporte vital como la atención en Unidades de Cuidados Intermedios, la Ventilación Mecánica Invasiva (VMI), la Oxigenación por Membrana Extracorpórea (ECMO) o la diálisis en estados agudos, así como otros medios o procedimientos que busquen sostener la vida y mejorar la posibilidad de supervivencia, las cuales para efectos del presente documento se denominarán Soporte Vital Avanzado (SVA), se prevé una difícil situación, pues las proyecciones de los modelos matemáticos disponibles muestran que, es probable que el número de casos que requerirán este tipo de soporte superará la oferta disponible durante los picos de la pandemia. ${ }^{\mathrm{I}}$

Dentro del marco de la declaración de pandemia por COVID-I9 por parte de la Organización Mundial de la Salud, el Gobierno colombiano

1 La estimación de la OMS, con base en las estadísticas de Wu Han en 2020, señala que un 6,1\% de los pacientes diagnosticados con COVID-19 requerirán atención en unidad de cuidado intensivo. declaró un estado de emergencia sanitaria, con el objeto de dar vía a la asignación excepcional de recursos públicos para responder a la emergencia por COVID-I9, y como medida anticipatoria y preparatoria, ha hecho un esfuerzo por incrementar la oferta de camas de UCI y de equipos de VMI. Al mismo tiempo el Gobierno ha recomendado al sector prestador que, en la medida de lo posible, se liberen los recursos existentes para mejorar la disponibilidad de estos durante los picos de demanda, así como les ha recomendado contar con reglas claras para permitir el triaje organizado por niveles de cuidado a través de recomendaciones específicas o protocolos estandarizados para los profesionales.

A pesar de tales esfuerzos, y teniendo en cuenta el comportamiento que ha mostrado la pandemia por COVID-I9 en otros países, se espera que, inevitablemente, en algunas instituciones prestadoras, durante algunos espacios de tiempo, los picos de demanda superen la oferta disponible de recursos tecnológicos y humanos para el SVA. Ante una circunstancia de oferta insuficiente para responder a la demanda aumentada, se hace necesario recurrir a una lógica basada en el principio de justicia distributiva, según el cual se debe maximizar el resultado agregado en salud para la sociedad. Esto supone que será necesario, solo de manera excepcional, y cuando se demuestre escasez, dar prioridad al beneficio colectivo sobre el beneficio individual. Dentro del marco de derechos humanos, la protección del derecho fundamental a la salud en el contexto de la emergencia se entiende como el acceso a la atención sanitaria de máximo nivel que pueda proporcionarse, buscando el grado máximo de salud que se pueda lograr.

Para hacer operativa esta lógica, se debe aplicar un proceso de triaje, el cual consiste en utilizar criterios específicos para priorizar la asignación del recurso escaso según la probabilidad que un paciente dado tenga de obtener resultados exitosos en salud cuando se beneficia del recurso en cuestión, para este caso específico, el SVA. Esta priorización implica, inevitablemente, que otro $u$ otros pacientes no 
podrán beneficiarse de la asignación de este recurso escaso.

El proceder ético del médico frente al SVA, por fuera del escenario de crisis, está basado en buscar el beneficio para el paciente, sin tener en cuenta las consecuencias distributivas de tales decisiones. La modificación de este proceder ético, para dar prelación al principio de justicia distributiva en las circunstancias excepcionales de la emergencia, genera una gran carga moral y emocional en el médico que se ve obligado a tomar este tipo de decisiones. Cabe anotar que esta carga moral y emocional no se limita a los médicos, sino que también impacta a otros profesionales de la salud, y a la sociedad en general, para quienes puede ser especialmente difícil aceptar que sea imposible salvar todas las vidas.

Ante esta retadora situación, se hace necesario establecer unas recomendaciones para enfrentar las decisiones de triaje de SVA, de tal manera que estas sean vistas como válidas, justas, eficaces y transparentes para que gocen de la mayor legitimidad posible, y que además sean imparciales y equitativas. Estas recomendaciones tienen como objetivos: I) ayudar a preservar la confianza de la sociedad en el proceder ético del médico en estas circunstancias excepcionales; 2) mejorar la aceptación social de la inescapable realidad de la limitación de recursos tecnológicos y humanos durante los picos de demanda de SVA; 3) reducir la carga moral y emocional del médico que se ve obligado a realizar triaje de SVA; y 4) contribuir al desarrollo de la lex-artis en la situación excepcional que obliga a tomar decisiones de triaje de SVA.

Por último, cabe resaltar que las recomendaciones de este documento son aplicables solamente dentro del contexto de la emergencia sanitaria declarada por el gobierno nacional, a causa de la pandemia de COVID-I9. Su alcance solamente se refiere a la decisión de asignar recursos para SVA para pacientes de COVID-I9 y para pacientes con otras condiciones que lo requieran, en las circunstancias en las que la demanda de SVA supera los recursos disponibles. Aunque la pandemia de COVID-I9 genera otros dilemas éticos frente a asuntos como el uso de tratamientos experimentales, la restricción a las libertades individuales, entre otros, este documento solo se enfoca en los dilemas éticos asociados al SVA.

\section{Principios}

Justicia distributiva: Cuando los recursos son insuficientes para satisfacer las necesidades de las personas, su distribución debe partir de la base de buscar el mayor bienestar agregado posible. Este principio obliga a que, en la elección de pacientes para SVA en esta situación excepcional, se busque maximizar el bienestar agregado. En esta situación de excepción el principio de justicia distributiva tendrá prelación sobre el principio de beneficencia, pero siempre respetando la dignidad humana y protegiendo el derecho fundamental a la salud al máximo nivel que sea factible de proporcionar, dada la insuficiencia del recurso para SVA.

Autonomía del paciente: Como medida de respeto a las personas, todo paciente tiene el derecho a elegir: I) si quiere o no participar en la toma de decisiones y 2) si recibe o no los tratamientos disponibles, después de haber recibido información sobre los riesgos y los beneficios, expresando en consecuencia su libre e inequívoco consentimiento para la intervención o el rechazo de la misma. Para proteger la autonomía de las personas se deberá siempre proveer información transparente y objetiva, incluyendo información concreta sobre la posibilidad de que en condiciones de escasez y de necesidad de realizar triaje para SVA, sus preferencias y derechos individuales puedan verse limitados. Salvo en las situaciones de excepción previstas en el marco regulatorio, se preservará el deber de informar al paciente y se respetará la autonomía del paciente para aceptar o rechazar tratamientos.

Beneficencia: A pesar de que, en un contexto de emergencia y de exceso de demanda, es aceptado realizar triaje, se debe buscar el mejor beneficio para todos los pacientes en cada una de las posibilidades de atención disponibles. Esto implica que, para cada paciente, respetando su 
autonomía para aceptar o rechazar tratamientos, se debe definir: I) el nivel de cuidado requerido, tanto para el inicio de SVA como para su suspensión en caso de que sea necesario; 2) el cumplimiento o no de los criterios de elegibilidad para SVA; y 3 ) inicio de medidas paliativas en caso de no iniciar o de suspender el SVA.

No maleficencia: Tanto en los pacientes en los que se inicie SVA como en los que no se inicie o les sea suspendido, las acciones disponibles se ponderarán en términos de no causar daño a los pacientes ni allegados. Esto incluye no abandonar el cuidado y actuar respetando el tipo de cuidado asignado de acuerdo con criterios objetivos. El no iniciar SVA o el suspenderlo, cuando, en circunstancias de no emergencia, el paciente podría haber tenido la oportunidad de recibirlo, no se debe entender como una forma de causar daño, sino como una forma de buscar un beneficio agregado mayor. Tomar decisiones con base en la mejor evidencia disponible es una forma de evitar el daño, pero dado que la evidencia sobre COVID-I9 se modifica con mayor frecuencia de lo habitual, las conductas médicas deben conservar la flexibilidad necesaria para ajustarse a esta evidencia en rápida evolución. Adicionalmente, esta ausencia de evidencia concluyente debe servir para llevar las expectativas de la sociedad sobre probabilidad de contagio, probabilidad de curación y probabilidad de muerte, a su correcto nivel y evitar falsas expectativas.

Respeto a la dignidad humana: Todas las acciones de cuidado integral de las personas, independientemente de la circunstancia de emergencia, se deben realizar y garantizar en el marco del respeto de su dignidad humana y los derechos humanos, por lo que los pacientes y sus familiares siempre serán reconocidos como personas independientemente de cualquier otra característica o rasgo individual.

Proporcionalidad: Este principio establece que las medidas terapéuticas que se definan deben mostrar una razonable relación entre los medios empleados, y el fin previsible para el paciente. Es decir, no es justificable realizar acciones heroicas en la atención de un paciente que requiere SVA si no existe una probabilidad razonable de que el paciente se recuperará de su estado agudo.

Utilidad: El principio de utilidad establece que las acciones son correctas en la medida que promueven el bienestar de individuos o comunidades. Los esfuerzos para maximizar la utilidad requieren consideraciones de proporcionalidad para equilibrar los beneficios potenciales de cualquier actividad frente a cualquier riesgo o daño resultado de esta, y tienen en cuenta la eficiencia para lograr los mayores beneficios al menor costo posible.

Equidad: La distribución de los recursos para el SVA debe buscar que aquellas personas con mayores necesidades reciban más que aquellas con menores necesidades. Sin embargo, en las condiciones excepcionales de emergencia, la escasez de recursos es más crítica y es posible que, ante la necesidad de realizar triaje, no se puedan satisfacer estas necesidades plenamente a algunas personas.

Imparcialidad y no discriminación: En consonancia con el respeto a los derechos humanos, y en reconocimiento a la dignidad de todas las personas, ninguna persona perteneciente a una población vulnerable de la sociedad debe tener un chance inferior de ser seleccionada para SVA que otra persona en condiciones similares, pero no perteneciente esta. La edad por sí sola no debe ser un criterio de decisión, sino el estado de salud general del paciente y su probabilidad de recuperación. Así como la edad, la presencia de cualquier condición de discapacidad no se considera un criterio exclusivo de no inicio ni de suspensión de SVA.

Transparencia: Los principios, criterios y recomendaciones que se aplicarán en la toma de decisiones de triaje, se hacen explícitos en este documento. Las decisiones de triaje se tomarán siguiendo los principios, criterios y recomendaciones que aquí se plantean.

\section{Recomendaciones}

Con base en los principios enunciados y teniendo en cuenta el contexto y las circunstancias 
excepcionales de la emergencia por COVID-I9, a continuación se plantean las recomendaciones para enfrentar los conflictos éticos que emergen en la asignación de recursos limitados para SVA.

\section{Recomendaciones de justicia procedimental}

Con el fin de que el resultado de la asignación de recursos limitados para SVA sea aceptado como legítimo, tanto por la sociedad en general como por los pacientes afectados, sus familiares y otras personas relevantes, se debe garantizar que la definición de las recomendaciones del presente documento y el proceso para la toma de decisiones sean imparciales. Para este fin se deben cumplir los siguientes atributos:

Rendición de cuentas: Tanto las recomendaciones del presente documento como su aplicación en la vida real deben estar disponibles para el escrutinio público y de las instituciones profesionales.

Publicidad: Los principios, criterios y recomendaciones de este documento se deben poner a disposición del público para que este conozca de su existencia y delibere sobre su pertinencia y aceptabilidad. Aunque no se espera que en una situación de triaje haya deliberación, pues la situación de urgencia obliga a tomar decisiones de manera rápida, sí se espera que haya deliberación en espacios diferentes a la línea del frente.

Razonabilidad de los principios: Los principios establecidos son razonables, y por tratarse de una situación excepcional de emergencia causada por la COVID-I9, su aplicación y su ordenamiento jerárquico son razonables. En condiciones de no emergencia alguno de estos principios, su aplicación y su ordenamiento jerárquico, pueden ser diferentes.

Apelaciones: Los grupos de interés pueden manifestar sus diferencias con estas recomendaciones y se deben abrir espacios de deliberación que permitan fortalecer la confianza de la sociedad en la profesión médica y en la respuesta del gobierno a la emergencia. Esta deliberación no debe ocurrir en la línea del frente sino en otros espacios por fuera de esta.
Conflictos de intereses: El médico no tomará decisiones de triaje para favorecer familiares o personas relevantes a sus intereses personales. En estos casos la decisión debe ser tomada por otro médico, excepto si no hay otro médico disponible. Tampoco tomará decisiones basadas en recomendaciones administrativas o de terceros.

\section{Recomendaciones generales para la toma de decisiones}

Ningún principio o criterio deberá ser usado de forma aislada. Estos principios y criterios deben ser considerados de manera conjunta, y deben ser siempre ponderados de acuerdo a la mejor evidencia disponible, actuando con flexibilidad y adaptabilidad a la nueva evidencia disponible para garantizar el mejor cuidado y atención posible. En consecuencia, se listan las siguientes recomendaciones generales sobre la decisión de realizar triaje para SVA:

I) Definir criterios de activación y desactivación del triaje. Dado que las condiciones de oferta y demanda en un hospital dado cambian en el tiempo, es necesario definir los criterios para activar el triaje para SVA cuando la demanda supere la oferta, y para desactivarlo cuando deje de existir esta situación. Dentro de cada institución prestadora, las decisiones de activar y desactivar el triaje deben quedar formalizadas para delimitar con precisión las condiciones de excepcionalidad durante las cuales se aplicarán las recomendaciones relacionadas con el triaje. Cada institución prestadora deberá definir con precisión este formalismo.

2) Antes de activar el triaje en una institución prestadora, en la medida de lo posible se debe procurar trasladar pacientes a otras instituciones prestadoras mediante los mecanismos de coordinación local instituidos. Lo anterior teniendo en cuenta la utilidad o futilidad del traslado según la severidad del caso y la probabilidad de deterioro durante este. Adicionalmente, para adelantar el traslado debe tenerse en 
cuenta que ello no implique dejar a la institución remisora sin el recurso humano y tecnológico que requiere para atender sus necesidades locales.

3) Rechazar el criterio de asignación por "orden de llegada". Este criterio es injusto y no imparcial, puesto que favorece a las personas que viven cerca a las instituciones prestadoras, o que enfrentan menos barreras de acceso para llegar a estas, y no permite aplicar los principios enunciados previamente en especial el principio de justicia distributiva.

4) No aplicar el criterio de valor social del paciente. En una sociedad plural no es posible afirmar que una persona sea intrínsecamente más valiosa que otra; en consecuencia, el valor social percibido de una persona no será un criterio de decisión.

5) Tener en cuenta el valor instrumental de los trabajadores de la salud de la línea del frente. Esta recomendación se basa en dos elementos: i) Las habilidades y capacidades de estos son requeridas para enfrentar la emergencia, es decir, si enferman o fallecen, habrá menos trabajadores de la salud para tratar al resto de personas enfermas; $y$ ii) el principio de reciprocidad, según el cual, al estar expuestos a un riesgo desproporcionado para enfrentar una amenaza colectiva, la sociedad les debe dar un tratamiento especial que compense dicha exposición al riesgo.

6) Tener en cuenta que las decisiones de selección de pacientes no se basan en criterios absolutos que se apliquen en todas las circunstancias, sino que son relativos a los demás pacientes que en un momento dado compiten por la asignación del SVA sean pacientes con COVID-I9 o con condiciones médicas diferentes a COVID-I9 que requieran SVA. Esto en razón a que las circunstancias de insuficiencia de oferta y las características de los pacientes elegibles para SVA son diferentes entre una institución prestadora y otra, o entre momentos distintos en una misma institución prestadora.

7) Procurar que la toma de decisiones se haga de manera colegiada. En la medida de lo posible, se debe evitar que un médico tome individualmente la decisión de no iniciar o de suspender SVA en pacientes que, siendo elegibles para este, quedan excluidos al aplicar el triaje. Este tipo de decisiones generan una alta carga moral y emocional en el médico, por lo cual debe: i) buscar apoyo de los Comités de Ética Hospitalaria allí donde se cuente con estos; ii) si no se cuenta con estos comités, debe buscar el apoyo de una segunda opinión con otro médico de la institución; iii) en caso de que esto no sea posible, debe buscar el apoyo de una segunda opinión por fuera de la institución.

8) Las recomendaciones incluidas en este documento deben ser operacionalizadas en detalle en las instituciones prestadoras, de acuerdo con los lineamientos y normas de las autoridades de salud y del gobierno en general.

\section{Recomendaciones específicas para la realización del triaje para SVA}

En condiciones de no emergencia, existen unos criterios convencionales de elegibilidad para SVA que son basados en evidencia y que son generalmente aceptados por la profesión médica y por la sociedad. Estos criterios están definidos en función de la probabilidad de recuperación del paciente en el corto plazo sin que requiera SVA una vez superado el episodio agudo, y teniendo en cuenta el consentimiento del paciente o su representante. Sin embargo, en una situación de oferta insuficiente de SVA, es necesario adicionar otros criterios generales, puesto que el aplicar los criterios convencionales de elegibilidad puede dar como resultado que haya muchos más pacientes elegibles que recursos de SVA disponibles.

En consecuencia, se listan las siguientes recomendaciones para la realización del triaje para SVA: 
I) Aplicar inicialmente los criterios convencionales de elegibilidad para SVA. ${ }^{2}$ Estos criterios se basan en mediciones objetivas y verificables y se deben aplicar en función de la probabilidad de recuperación del paciente en el corto plazo sin que requiera SVA una vez superado el episodio agudo. Un criterio de no elegibilidad para SVA es la decisión del paciente de no aceptarlo.

2) En caso de que, después de aplicar los criterios convencionales de elegibilidad para SVA, haya más pacientes elegibles que recurso disponible, la elección se debe basar en el análisis simultáneo de los siguientes cuatro criterios: (i) pronóstico de supervivencia a corto plazo, dados los factores predictores de supervivencia específicos para COVID-I9 que se hayan identificado en la literatura científica; estos factores predictores deben ser actualizados periódicamente en la medida que la nueva evidencia científica sobre COVID-I9 así lo requiera33; (ii) pronóstico de supervivencia a largo plazo o mayor expectativa de vida; (iii) presencia de comorbilidades que afecten el pronóstico de largo plazo; y (iv) qué tantos recursos de SVA se espera que consumirá el paciente.

3) La consideración de manera simultánea de los cuatro criterios enunciados en el numeral 2) implica que ninguno de ellos se debe aplicar de manera aislada, no se deben aplicar de manera secuencial, y solo deben aplicarse después de aplicar los criterios convencionales de elegibilidad para SVA, como lo indica el numeral I).

\section{Recomendaciones para un actuar ético en el manejo del paciente que será tratado en la UCI}

Una vez se ha definido que el paciente es elegible para el ingreso a la UCI, es necesario verificar

2 Se deben consultar los criterios establecidos por la Asociación Colombiana de Medicina Crítica y Cuidado Intensivo (AMCI).

3 Se deben consultar los criterios establecidos por la Asociación Colombiana de Infectología (ACIN). que el tratamiento instaurado esté logrando el beneficio esperado, pues de no lograrse, y en caso de haber exceso de demanda, se debe considerar la posibilidad de suspenderlo. Tanto la decisión de iniciar como la de suspender tratamiento, deben respetar la autonomía del paciente, dentro de los términos establecidos por la legislación y jurisprudencia colombianas. En consecuencia, se listan las siguientes recomendaciones para el actuar ético en la UCI:

I) A todo paciente a quien se le inicia SVA se le debe diseñar un plan de manejo con metas explícitas basadas en parámetros biomédicos y los tiempos esperados de logro de estas metas. Este plan de manejo debe respetar la autonomía del paciente para aceptar o rechazar las medidas de SVA, autonomía que se debe materializar, en la medida de lo posible, en el consentimiento informado y en el Documento de Voluntad Anticipada.

2) El plan de manejo debe ser revisado cada 24 horas y si el paciente no logra las metas establecidas, se debe considerar la suspensión del SVA y el inicio de la adecuación de los esfuerzos terapéuticos. La suspensión no es justificable éticamente con el fin de tener oferta disponible de SVA ante una mera expectativa de pacientes futuros.

3) Salvo las excepciones al deber de informar que se consideran en el marco regulatorio vigente, se debe dar información de forma temprana y continua al paciente o a su representante, con el propósito de garantizar la comunicación sobre las preferencias individuales frente a las atenciones específicas que se pueden ofrecer y sobre el curso de la enfermedad. Lo anterior debe incluir información sobre el plan de manejo completo y la posibilidad de que sea necesaria la adecuación del esfuerzo terapéutico.

4) Cuando el exceso de demanda es tal que no es posible establecer comunicación efectiva con el paciente y/o con su representante y/o con sus familiares debido al escaso tiempo 
disponible para entrar en este tipo de comunicaciones, el médico tomará las decisiones que sean pertinentes en ejercicio de su capacidad técnica con responsabilidad y proporcionalidad, y las registrará posteriormente en la historia clínica.

5) Salvo las excepciones al deber de informar que se consideran en el marco regulatorio vigente, en el momento del ingreso al hospital se debe solicitar información al paciente o a su representante sobre la existencia de Documentos de Voluntad Anticipada o el deseo de suscribir uno como medida de autogobierno y ejercicio de derechos. Estos documentos deben enfocarse en la aceptación de medidas de soporte avanzado progresivamente o de rechazo de las mismas, con particular atención a los pacientes que por deterioro del estado general pudieran tener alteraciones cognitivas o requerir aislamiento y en quienes se evidencia una discrepancia entre sus preferencias y las de su representante o familiares.

6) Cuando el paciente ha expresado voluntariamente mediante consentimiento informado o Documentos de Voluntad Anticipada su rechazo a ser ingresado a UCI o a recibir SVA, esta decisión debe ser respetada, independientemente del pronóstico que tenga el paciente en el momento de enfrentar la decisión de ingreso a UCI o de inicio de SVA.

7) Salvo las excepciones al deber de informar que se consideran en el marco regulatorio vigente, se debe informar al paciente sobre la potestad que tiene de rechazar las terapias, en cuyo caso debe informarse sobre el posible beneficio del uso de SVA y las alternativas disponibles, y registrar de forma clara en la historia clínica que el paciente rechaza de forma directa el inicio de los soportes, indicando los soportes rechazados y la comprensión de las consecuencias de la no instauración de estos.

8) En caso de usar consentimientos informados escritos o documentos de rechazo terapéuti- co, su uso debe ser claro y consistente para todos los pacientes, y deben realizarse en el momento de la atención con un proceso consecuente con la recepción de información clara y objetiva. Ambos son documentos que firma la persona que se encuentra como paciente. Debe tenerse en cuenta que en un consentimiento sustituto no se pueden rechazar medidas útiles.

9) Debe tenerse en cuenta que la adecuación de los esfuerzos terapéuticos no requiere ningún tipo de documento o formalismo diferente al registro suficiente en la historia clínica sobre la conducta. La adecuación de los esfuerzos terapéuticos es una conducta médica, y por lo tanto es el médico quien, en ejercicio de su capacidad técnica con responsabilidad y proporcionalidad, define en qué circunstancias un paciente no se beneficia de un medio, procedimiento o tratamiento. La decisión de adecuación de los esfuerzos terapéuticos no se debe transferir al paciente o los familiares, aunque estos pueden solicitarla de forma activa como forma del ejercicio del derecho a morir con dignidad. Si bien, la adecuación de los esfuerzos terapéuticos hace parte de las formas del ejercicio del derecho a morir con dignidad para evitar la obstinación terapéutica y la prolongación biológica de la vida por medio de intervenciones fútiles, en el escenario de COVID-I9 se incluye como medida inicial ante la posibilidad de que no haya indicaciones para el inicio de SVA, o ante la circunstancia excepcional en la que por escasez del recurso no sea posible iniciar el SVA o sea necesaria su suspensión.

Io) En cuanto a la aplicación de intervenciones experimentales, dentro del plan de manejo del paciente y durante el período de excepción de la emergencia sanitaria, se podrán considerar este tipo de intervenciones sin que necesariamente estén enmarcadas dentro de un ensayo clínico, siempre y cuando se cumplan las siguientes condiciones: i) que no exista una alternativa terapéutica efectiva 
comprobada, o que hayan fallado las alternativas disponibles; ii) que no sea posible iniciar un estudio clínico inmediatamente; iii) que haya datos disponibles que apoyen de manera preliminar la eficacia y seguridad de la intervención, al menos basados en estudios de laboratorio en animales, o que su uso por fuera de estudios clínicos haya sido sugerido por las sociedades científicas de la profesión médica; iv) que cuenten con aprobación del Invima para su uso experimental, y la aprobación de un comité de ética; v) que se garanticen los recursos adecuados para minimizar los riesgos de seguridad; vi) que se obtenga el consentimiento informado del paciente o su representante; vii) que se cuente con un sistema de monitorización de la intervención y que los resultados sean documentados y compartidos oportunamente con la comunidad médica y científica.

\section{Recomendaciones sobre el acompaña- miento y el manejo del final de la vida}

No es aceptable el abandono de un paciente por el hecho de haber llegado a la etapa del final de la vida. La garantía del alivio sintomático y el acompañamiento al proceso de final de la vida hacen parte del cuidado integral de los pacientes. En consecuencia, se listan las siguientes recomendaciones para el acompañamiento y el manejo de final de la vida:

I) Se debe revisar permanentemente la necesidad de alivio sintomático según la evolución clínica del paciente, específicamente en términos de control del dolor y sufrimiento secundarios a la presencia de disnea, secreciones respiratorias, tos, ansiedad y presencia de delirium, u otros síntomas propios de la condición médica del paciente. Los cuidados de final de la vida se deben garantizar a todos los pacientes que los requieran, independientemente de que se disponga o no de equipos humanos especializados en cuidados paliativos.

2) En caso del paciente que manifiesta su decisión de rechazo terapéutico, cuando su deterioro sea previsible como consecuencia de dicha manifestación, se debe trasladar al paciente a un espacio diferente a la UCI.

3) Informar al paciente y a sus familiares que, en caso de fallecimiento del paciente, el acompañamiento al cadáver se hará estrictamente bajo los protocolos establecidos por las autoridades de salud pública, debido al alto riesgo de contagio para los acompañantes.

4) Se deben garantizar medidas especiales de apoyo psicológico para los familiares y acompañantes del fallecido, dadas las circunstancias excepcionales de aislamiento del cadáver, que no permiten la realización del duelo como se haría en circunstancias de no emergencia sanitaria.

\section{Recomendaciones sobre la protección y cuidado de los trabajadores de la salud}

Los trabajadores de la salud, especialmente aquellos que están en la línea del frente, es decir, en contacto con pacientes con COVID-I9, enfrentan un riesgo mayor de contagio de la enfermedad en comparación con el resto de la población, por lo cual se deben tomar medidas especiales para su protección frente a este riesgo. Adicionalmente, es necesario reducir al mínimo posible el riesgo de contagio del personal de la línea del frente pues en la medida en que adquieran la enfermedad o fallezcan como consecuencia de esta, su ausencia temporal o permanente incrementará el riesgo de que la demanda supere los recursos disponibles.

I) A los médicos y demás trabajadores de la salud de la línea del frente se les deben suministrar los recursos necesarios para reducir la exposición al riesgo de contagio por $\mathrm{CO}$ VID-I9 durante sus actividades laborales, específicamente: i) Elementos de protección personal, según el tipo de exposición al riesgo de contagio; y ii) adecuado entrenamiento, según los roles a desempeñar durante la emergencia, para reducir el riesgo de contagio. 
2) En caso de no contar con los elementos de protección personal adecuados, el médico podrá rehusarse a prestar los servicios de atención a pacientes con COVID-I9.

3) Fuera del contexto de la línea del frente, el personal de salud tiene las mismas obligaciones que el resto de la sociedad, en relación con el distanciamiento físico, medidas de higiene, uso de mascarillas, y las demás que establezca el gobierno.

4) Adicionalmente, se deben definir los criterios de protección del personal de salud en las situaciones en las que se requiera resucitación cardiopulmonar, especialmente cuando los riesgos de exposición al contagio del personal de salud sean mayores que los beneficios esperados de la resucitación.

5) Se debe contar con medidas de apoyo sicológico para manejar el estrés del personal de la línea del frente, causado por el temor al contagio, la sobrecarga de trabajo y la carga moral y emocional de tener que negar a pacientes el acceso a SVA.

6) Los trabajadores de la salud con factores de riesgo de mayor severidad de COVID-I9, según la mejor evidencia disponible, no deben estar expuestos al contacto directo con pacientes de COVID-I9.

\section{Agradecimientos:}

El equipo de trabajo agradece las contribuciones de las siguientes instituciones y personas:

- Instituto Colombiano de Estudios Bioéticos, en especial a los doctores Fernando Sánchez Torres, Edgar Montoya Ángel, Rosemary León Buitrago, Ricardo Roa Castellanos, Gonzalo D. Prada González, Elena Rey Lozano, Fabio Raúl Trompa Ayala, Benjamín Herazo Acuña, Carlos Gaviria Neira, Alejandro Castaño Bedoya, y Luis María Murillo.

- Grupo de Investigación en Bioética y Bioderecho de la Universidad del Rosario, en especial a los doctores Ana Isabel Gómez Córdoba, Piedad Bolívar Goez, Boris Julián Pinto Bustamante, Diana Bernal Camargo y Carlos Enrique Trillos Peña.

- Al doctor Maurizio Bonati, del Istituto di Ricerche Farmacologiche Mario Negri.

\section{Cumplimiento de normas éticas:}

Elaborado y avalado por: Equipo de trabajo de la Academia Nacional de Medicina de Colombia.

Consentimiento informado: Esta publicación es una revisión de la literatura, y como tal no hay necesidad de un consentimiento informado ni de aprobación del Comité de Ética Institucional.

Conflicto de intereses: Ninguno declarado por los autores.

\section{Referencias}

Los siguientes documentos sirvieron como referencia para construir el marco ético de los principios y las recomendaciones:

Berlinger N, Wynia M, Powell T, Hester M, Milliken A, Fabi R, et al. Ethical framework for health care institutions and guidelines for institutional ethics services responding to the novel coronavirus pandemic. The Hastings Center. 2020. Disponible en:

https://www.thehastingscenter.org/ethicalframeworkCOVIDig/

- Biddison ELD, Faden R, Gwon HS, Mareiniss DP, Regenberg AC, Schoch-Spana M, et al. Too many patients...A framework to guide statewide allocation of scarce mechanical ventilation during disasters. Chest. 20I9;I55:848-854.

https://doi.org/IO.IOI6/j.chest.2018.09.025

Comité Internacional de Bioética de la UNESCO y Comisión Mundial de Ética del Conocimiento Científico y la Tecnología de la UNESCO. Declaración sobre el COVID-I9: Consideraciones éticas desde una perspectiva global. 2020. Disponible en:

https://unesdoc.unesco.org/ark:/48223/pfoooo373115_spa

Dunham AM, Rieder TN, Numbyrd C. A bioethical perspective for navigating moral dilemmas amidst the COVID-I9 pandemic. J Am Acad Orthop Surg. 2020. https://doi.org/IO.5435/JAAOS-D-20-0037I 
- $\quad$ Emmanuel E, Persad G, Upshur R, Thome B, Parker M, Glickman A, et al. Fair allocation of scarce medical resources in the time of COVID-I9. N Engl J Med. 2020;382:2049-2055.

https://doi.org/IO.IO56/NEJMsb2005II4

- $\quad$ Truog RD, Mitchell C, Daley GQ. The toughest triage: Allocating ventilators in a pandemic. N Engl J Med. 2020;382:1973-1975.

https://doi.org/IO.IO56/NEJMp2005689

- White DB, Katz MH, Luce JM, Lo B. Who should receive life support during a public health emergency? Using ethical principles to improve allocation decisions. Ann Intern Med. 2009;150:I32-138.

- White DB, Lo B. A Framework for rationing ventilators and critical care beds during the COVID-I9 pandemic. JAMA. 2020;323:I773-4. https://doi.org/IO.IOoI/jama.2020.5046

- World Health Organization. Report of the WHO-China joint mission on coronavirus disease 2019 (COVID-I9). 2020. Disponible en:

https://www.who.int/publications-detail/reportof-the-who-china-joint-mission-oncoronavirus-disease2019-(COVID-19)

- World Health Organization. WHO [Internet]. Guidance for managing ethical issues in infectious disease outbreaks. 20I6. [Fecha de acceso] Disponible en: https://apps.who.int/iris/handle/I0665/250580

Adicionalmente, se consultaron las siguientes declaraciones emitidas por organismos públicos y por asociaciones médicas para identificar los temas a incluir y la estructura general de las recomendaciones:

\section{Colombia}

- Accini-Mendoza JL, Beltrán V, Nieto-Estrada H, Ramos-Bolaños E, Pizarro-Gómez C, Rebolledo CE, et al. Declaración de consenso en medicina crítica para la atención multidisciplinaria del paciente con sospecha o confirmación diagnóstica de COVID-19. Acta Colomb Cuid Intensivo. 2020. (en prensa). https://doi.org/IO.IOI6/j.acci.2020.04.003

- Grupo ACIN- IETS de Consenso Colombiano para recomendaciones de atención COVIDi9. Consenso Colombiano de atención, diagnóstico y manejo de la infección por SARS-COV-2/COVID-I9 en establecimientos de atención de la salud. Recomendaciones basadas en consenso de expertos e informadas en la evidencia. Infectio. 2020;24(3)Supl:I-I53. http://dx.doi.org/Io.22354/in.v24i3.85I

- Ministerio de Salud y Protección Social de Colombia. Recomendaciones generales para la toma de decisiones éticas en los servicios de salud durante la pandemia COVID-I9. 2020. Disponible en: https://www.minsalud.gov.co/Ministerio/Institucional/ Procesos\%20y\%2oprocedimientos/GIPSi3.pdf

\section{Alemania}

- DIVI. Entscheidungen über die Zuteilung von Ressourcen in der Notfallund der Intensivmedizin im Kontext der COVID-I9-Pandemie. Version 2. April 2020. Disponible en: https://www.divi.de/empfehlungen/publikationen/COVID-I9/I540-COVID-I9-ethik-empfehlung-v2/file

\section{Austria}

- $\quad$ Bioethikkommission. Zum Umgang mit knappen Ressourcen in der Gesundheitsversorgung im Kontext der COVID-I9-Pandemie. März 2020. Disponible en: https://www.bundeskanzleramt.gv.at/dam/jcr:772d37bo3db9-4c8b-b4fe-e6dca7bib8d3/200402_COVID_Bioethik.pdf

\section{Chile}

- Sánchez I. Lineamientos éticos en la atención de pacientes en una situación de pandemia. Abril 2020. Disponible en:

https://medicina.uc.cl/noticias/lineamientos-eticos-en-la-atencion-de-pacientes-en-situacion-de-pandemia/

\section{España}

- $\quad$ Montalvo-Jääskeläinen F, Altisent-Trota R, Bellver-Capella V, Cadena-Serrano F, Reyes-López M, Gándara del Castillo A, et al. Informe del Comité de Bioética de España sobre los aspectos bioéticos de la priorización de recursos sanitarios en el contexto de la crisis del coronavirus. Marzo 2020. Disponible en:

http://assets.comitedebioetica.es/files/documentacion/ Informe\%20CBE-\%2oPriorizacion\%2ode\%2orecursos\%2Osanitarios-coronavirus\%2oCBE.pdf

- Grupo de Trabajo de Bioética de la Sociedad Española de Medicina Intensiva, Crítica y Unidades Coronarias. Recomendaciones éticas para la toma de decisiones en la situación excepcional de crisis por pandemia COVID-I9 en las Unidades de Cuidados Intensivos Semicyuc. 2020. Disponible en: https://semicyuc.org/wp-content/uploads/2020/03/\%C3\%89tica_SEMICYUC-COVID-I9.pdf 


\section{Estados Unidos}

- Estado de Alabama. Criteria for mechanical ventilator triage following proclamation of mass-casualty respiratory emergency. 20I0. Disponible en:

https://adap.ua.edu/uploads/5/7/8/9/57892I4I/alabamas_ventilator_rationing_plan.pdf

\section{Francia}

- Comité Consultatif National d'Éthique. Contribution du Comité Consultatif National d'Éthique: Enjeux Éthiques Face à une Pandémie. 2020. Disponible en: https://www.ccne-ethique.fr/sites/default/files/reponse_ccne_-_COVID-I9_def.pdf

\section{Italia}

- $\quad$ SIAARTI. Raccomandazioni di Etica Clinica per L'ammissione a Trattamenti Intensivi e per la Loro Sospensione, in Condizioni Eccezionali di Squilibrio tra Necessità e Risorse Disponibili. 2020. Disponible en: http://www.siaarti.it/SiteAssets/News/COVIDi9\%20 -\%20documenti\%2oSIAARTI/SIAARTI\%2O-\%20COVIDi9\%20-\%20Raccomandazioni\%2odi\%2oetica\%20 clinica.pdf

\section{México}

- Medina-Arellano MJ, Palacios-González C, Santos-Preciado JI. Guía bioética de asignación de recursos de medicina crítica del Consejo de Salubridad General en México. Salud Pública Mex. 2020;62:I-3.

https://doi.org/IO.2/II49/II747

\section{Reino Unido}

- $\quad$ British Medical Association. COVID-I9 - ethical issues. A guidance note. 2020. Disponible en: https:/www.bma.org.uk/media/2226/bma-COVID-I9ethics-guidance.pdf

Por último, el marco normativo relevante para este documento incluyó las siguientes normas:

- $\quad$ Congreso de Colombia. La Ley 23 de 198I. Disponible: https://docs.supersalud.gov.co/PortalWeb/Juridica/Leyes/Loo23_8I.pdf

- Presidencia de la República de Colombia. Decreto Número 3380 de 198I. Disponible:

http://www.suin-juriscol.gov.co/viewDocument.asp?ruta $=$ Decretos/I525998

- Ministerio de Salud y Protección Social de Colombia. Resolución Número 229 de 2020. Disponible en: https://www.minsalud.gov.co/Normatividad_Nuevo/ Forms/DispForm.aspx?ID=5903

- $\quad$ Ministerio de Salud y Protección Social de Colombia. Resolución Número Resolución 2665 de 2018 (25 de junio de 20I8). Disponible:

https://www.minsalud.gov.co/Normatividad_Nuevo/ Resoluci\%C3\%B3n\%20No.\%202665\%20de\%202018.pdf 